TITLE:

\title{
Theory of the Dielectric Dispersion due to the Interfacial Polarization and its Application to Emulsions
}

$\operatorname{AUTHOR}(S)$ :

Hanai, Tetsuya

\section{CITATION:}

Hanai, Tetsuya. Theory of the Dielectric Dispersion due to the Interfacial Polarization and its Application to Emulsions. Bulletin of the Institute for Chemical Research, Kyoto University 1961, 39(3): 249-249

ISSUE DATE:

1961-07-31

URL:

http://hdl.handle.net/2433/75832

RIGHT: 


\title{
Theory of the Dielectric Dispersion due to the Interfacial Polarization and its Application to Emulsions
}

\author{
Tetsuya Hanai \\ Kolloid Z., 171, 23 (1960)
}

The interfacial polarization of spherical dispersions is dealt with theoretically, and is discussed for the actual cases of emulsions.

First it is shown that the Maxwell theory is not valid qualitatively for the dielectric properties of emulsions. Next the general solution of the Wagner theory is derived. This solution shows that a dielectric dispersion due to the interfacial polarization is characterized by a single relaxation time. It is found that the Wagner theory is not valid quantitatively for the actual emulsions.

Finally a new theory of the interfacial polarization is developed on the assumption that the Wagner equation holds for an infinitesimally increasing process in concentration of the dispersion system. Several dielectric properties of emulsions are interpreted quanitatively by this new theory. Dielectric dispersions due to the interfacial polarization have so far bean observed only in the $\mathrm{W} / \mathrm{O}$ emulsions, and no example of the interfacial polarization has been found in usual emulsions of the $\mathrm{O} / \mathrm{W}$ type yet. According to the new theory it is suggested that, if the dielectric constant of the oil phase is much larger, the dielectric dispersion due to the interfacial polarization may be observed even in emulsions of the $\mathrm{O} / \mathrm{W}$ type.

\section{Dielectric Properties of Emulsions. (II) Electrical Conductivities of $\mathrm{O} / \mathrm{W}$ Emulsions}

Tetsuya Hanai, Naokazu Korzumi, Takeo Sugano and Rempei Gotoh

Kolloid Z., 171, 20 (1960)

In the previous paper (Kolloid Z., 16741 (1959)), dielectric constants of $\mathrm{O} / \mathrm{W}$ emulsions were reported. In this paper electrical conductivities of $\mathrm{O} / \mathrm{W}$ emulsions including $\mathrm{KCl}$ in the water phase were measured over a wide range of concentration and at frequencies ranging from $20 \mathrm{cps}$ to $3 \mathrm{mc}$.

No dielectric dispersion due to the interfacial polarization was observed in the experimental range of frequency, while the electrode polarization was observed over the present range of frequency.

Experimental results were compared with theoretical values for spherical dispersions. It was concluded that the electrical conductivities of $\mathrm{O} / \mathrm{W}$ emulsions were expressed fairly well by the equation of Bruggeman type over the whole range of concentration. 